\title{
Polarization equalization in optical vector network analysis for SDM fiber characterization
}

Rommel, Simon; van Weerdenburg, John; Mendinueta, Jose Manuel Delgado; Klaus, Werner; Sakaguchi, Jun; Vegas Olmos, Juan José; Koonen, Ton; Awaji, Yoshinari; Okonkwo, Chigo; Tafur Monroy, Idelfonso Total number of authors:

11

Published in:

IEEE Photonics Technology Letters

Link to article, DOI:

10.1109//pt.2019.2949615

Publication date:

2019

Document Version

Peer reviewed version

Link back to DTU Orbit

Citation (APA):

Rommel, S., van Weerdenburg, J., Mendinueta, J. M. D., Klaus, W., Sakaguchi, J., Vegas Olmos, J. J., Koonen, T., Awaii, Y., Okonkwo, C., Tafur Monroy, I., \& Wada, N. (2019). Polarization equalization in optical vector network analysis for SDM fiber characterization. IEEE Photonics Technology Letters, 31(24), 1917-1920. https://doi.org/10.1109/lpt.2019.2949615

\section{General rights}

Copyright and moral rights for the publications made accessible in the public portal are retained by the authors and/or other copyright owners and it is a condition of accessing publications that users recognise and abide by the legal requirements associated with these rights.

- Users may download and print one copy of any publication from the public portal for the purpose of private study or research.

- You may not further distribute the material or use it for any profit-making activity or commercial gain

- You may freely distribute the URL identifying the publication in the public portal 


\title{
Polarization equalization in optical vector network analysis for SDM fiber characterization
}

\author{
Simon Rommel, Member, IEEE, John van Weerdenburg, Student Member, IEEE, \\ José Manuel Delgado Mendinueta, Member, IEEE, Werner Klaus, Member, IEEE, Jun Sakaguchi, Member, IEEE, \\ Juan José Vegas Olmos, Senior Member, IEEE, Ton Koonen, Fellow, IEEE, Yoshinari Awaji, Member, IEEE, \\ Chigo Okonkwo, Senior Member, IEEE, Idelfonso Tafur Monroy, Senior Member, IEEE, and
} Naoya Wada, Member, IEEE

\begin{abstract}
Precise characterization of space division multiplexing fibers is a key requirement for their further development and deployment in the field. Optical vector network analysis allows to measure all linear parameters of such fibers in a single, fast acquisition over a large bandwidth. As the reference arm of the optical vector network analyzer must approximately match the length of the measured fiber, additional fiber must be introduced when measuring longer fibers. This results in the polarization of the reference arm varying with wavelength over the sweep range employed in the measurement. The resulting fading of the recorded interference signal produces severe distortions of the measurement and hence must be compensated for. This work introduces a simple equalization technique to compensate for this fading, based on introducing a polarizer in the reference path and recording a fraction of the polarized reference as a correction signal. Applying the inverse of the correction signal to the recorded signal, the impact of the polarization fading is almost entirely removed, as are the corresponding measurement distortions. The proposed technique is validated in the measurement of a 39-core few-mode multi-core fiber, significantly improving measurement of wavelength dependent insertion loss.
\end{abstract}

Index Terms-Optical vector network analysis, space division multiplexing, polarization, fiber characterization.

\section{INTRODUCTION}

$\mathbf{S}$ PACE division multiplexing (SDM) is a high potential approach to address the rapidly approaching limits of singlemode optical fiber communications systems [1], [2]. Recently, transmission rates beyond $10 \mathrm{Pbit} / \mathrm{s}$ have been demonstrated over a few-mode multi-core fiber (FM-MCF) [3]. Increasing

Manuscript received June 23, 2019; revised August 01, 2019, revised September 27, accepted Oct 21, 2019. This work was partially supported by the blueSPACE project which has received funding from the European Union's Horizon2020 research and innovation programme under grant agreement No. 762055 .

S. Rommel was with the Department of Photonics Engineering, Technical University of Denmark, 2800 Kgs. Lyngby, Denmark and with the Photonic Network System Laboratory, National Institute of Information and Communication Technology (NICT), 4-2-1 Nukui-Kitamachi, Koganei, Tokyo 1848795 , Japan. He is now with the Institute for Photonic Integration, Eindhoven University of Technology, 5600 MB Eindhoven, The Netherlands (e-mail: s.rommel@tue.nl).

J. M. Delgado Mendinueta, W. Klaus, J. Sakaguchi, Y. Awaji, and N. Wada are with the Photonic Network System Laboratory, National Institute of Information and Communication Technology (NICT), 4-2-1 Nukui-Kitamachi, Koganei, Tokyo 184-8795, Japan.

J. van Weerdenburg, T. Koonen, C. Okonkwo, and I. Tafur Monroy are with the Institute for Photonic Integration, Eindhoven University of Technology, 5600 MB Eindhoven, The Netherlands.

J. J. Vegas Olmos is with Mellanox Technologies, Ledreborg Allé 130B, 4000 Roskilde, Denmark spatial multiplicity, whether by exciting more modes or packing more cores in a single cladding, has its challenges and it appears that combing both approaches in FM-MCF yields the highest number of spatial channels and largest transmission capacities [3], [4]. One of the resulting challenges is managing spatial channel cross-talk, which requires multipleinput multiple-output (MIMO) digital signal processing (DSP) to unravel the mixing. The required number of dimensionsand hence the complexity of these algorithms-scales with the number of coupled spatial channels, while their required memory depth is dictated by the impulse response duration. Hence, a low differential mode group delay (DMGD), is desirable for strongly coupled spatial channels. Furthermore, variations among the gains of the spatial channels or mode dependent loss (MDL) degrade system capacity [5].

Accurate measurement methods and improved understanding of these impairments on both component and system level are required to support the rapid advancement of spacedivision multiplexing (SDM). Several techniques have been proposed to measure the impulse response of SDM fibers with high dynamic range [6], [7]. However, key parameters such as MDL cannot be derived from them. Optical vector network analyzer (OVNA) allows direct measurement of the complex transfer function matrix, from which all linear device parameters, such as insertion loss (IL), MDL, DMGD, and chromatic dispersion (CD), can be derived [8]-[12].

The operational principle behind the OVNA is swept wavelength interferometry (SWI), where a swept tunable source is guided through an interferometric structure which contains the device under test (DUT) in one of the arms. The frequency of the resulting fringe pattern is the product of the sweep rate and the interferometer path length differences, which is orders of magnitude lower compared to the observable optical bandwidth. Hence, relatively low speed photo-detectors and digitizers can be employed to achieve a high dynamic range. In order to maintain a small path length difference and thus keep the aforementioned advantages, an additional single-mode delay must be included in the reference arm for characterization of long fibers. During propagation through the single-mode reference fiber, the signal undergoes a polarization rotation. This rotation is observed to be wavelength dependent, resulting in power fading of the received signals which, if not properly compensated, will directly affect the calculated linear parameters. 


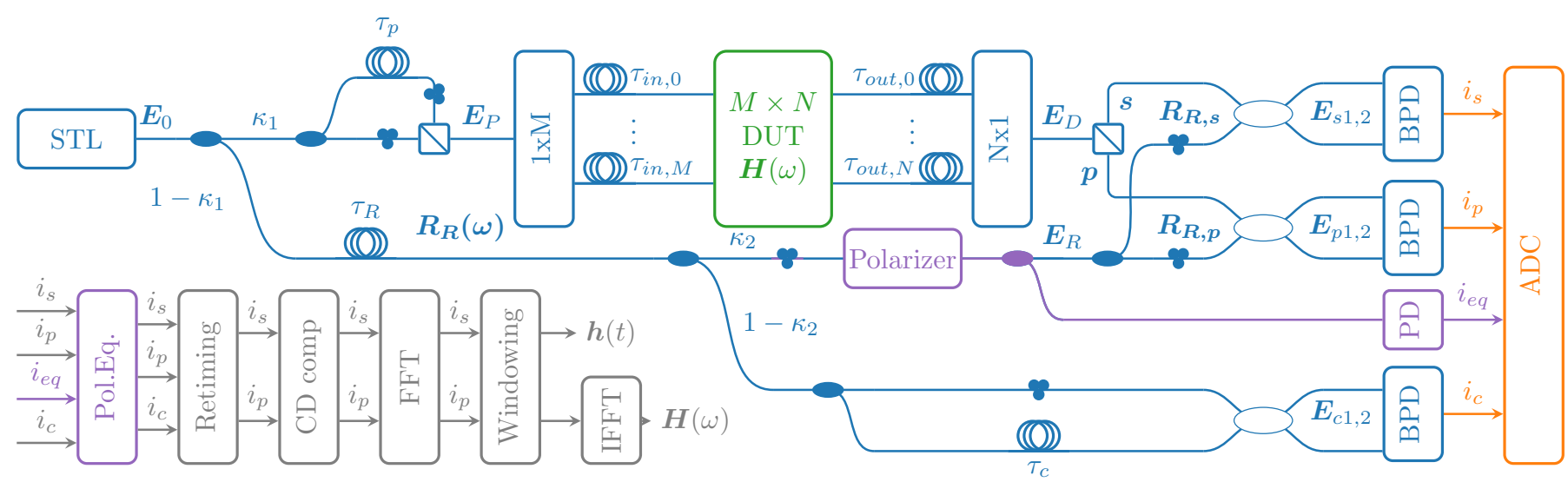

Fig. 1. OVNA setup for an $\mathrm{M} \times \mathrm{N}$ port device, including polarizer, detection of polarization reference and polarization equalization DSP step.

Expanding on our previous work on FM-MCF characterization with OVNA [11], [12], in this work we propose an equalization method that has minimum impact on the system setup, calibration and DSP complexity. The reference signal is polarized before it is combined with the light traversing trough the DUT. Furthermore, a fraction of the polarized reference is detected with an additional photodetector and sampled with the same digitizer. Before any other DSP steps are applied, the inverse of the tracker signal is employed to equalize the dual polarization measurement waveforms. Based on this method, we show that the fading arising from the wavelength dependency of the reference signal — which is directly evident in the IL extracted from the measurementcan be compensated in the measurement of a 39-core FMMCF. Showing a significantly reduced impact from polarization fading over wavelength, these measurements validate the proposed technique as a small but essential step in improving OVNA measurements of long lengths of fibers or in other circumstances where the polarization of the reference signal may vary with wavelength.

The remainder of this article is structured as follows: In Section II the issue of polarization fading in OVNA is introduced, followed by the proposed equalization in Section III. In Section IV the equalization method is applied to the characterization of a 39-core FM-MCF. In particular, the insertion losses over wavelength are discussed. The last section, Section V, concludes the article.

\section{Polarization Fading in Optical Vector NETWORK ANALYSIS}

A detailed and comprehensive derivation of the optical field at multiple points in the OVNA setup depicted in Fig. 1 is given in [11]. In that analysis it is assumed that the optical field $\boldsymbol{E}_{\mathrm{R}}$ of the reference arm at the input of the polarization diverse receiver can be aligned with the orthogonal polarization vectors $s$ and $\boldsymbol{p}$ of the polarization beam splitter (PBS) employed to split the field of the upper interferometer arm $\boldsymbol{E}_{\mathrm{D}}$ by a simple polarization controller (PC) in each arm. However, due to the fast wavelength tuning rate of the swept laser, this optimization can only be performed at a single wavelength.

Specifically, the polarization in the reference arm undergoes a rotation, described in [11] by a unitary $2 \times 2$ matrix $\boldsymbol{R}_{R}$, which, within a small optical bandwidth or for very short fiber lengths, can be presumed to be independent of the wavelength. In the case of analysis of longer fibers with an OVNA, where a length of fiber approximately matched to the fiber under test has to be added to the reference arm, this assumption no longer holds. In such a case, the output state of polarization will become wavelength dependent and thus a single wavelength optimization no longer suffices to align the signal from the reference with the fixed and wavelength independent polarization axes of the PBS in the device arm.

That is, the polarization rotation in the reference arm delay becomes wavelength dependent as $\boldsymbol{R}_{R}(\omega)$ and as a result a wavelength independent alignment $\boldsymbol{R}_{\mathrm{R}, s}$ (for the $s$ polarization arm of the PBS and polarization diverse receiver, analog for the $\boldsymbol{p}$ polarization arm) cannot ensure that reference and signal polarization are aligned at all wavelengths. The latter would result in severe wavelength dependent fading of the beat signal on the balanced photodetectors of the polarization diverse receiver.

The latter is easily seen, when regarding only the component of the reference signal $\boldsymbol{E}_{\mathrm{R}, s}$ co-polarized with the signal on the $s$ polarization arm (as only co-polarized signals will contribute to the beat pattern)

$$
\boldsymbol{E}_{\mathrm{R}, s}=\frac{1}{2} \sqrt{\frac{\kappa_{2}}{2}} \sqrt{\frac{1-\kappa_{1}}{2}} A \mathrm{e}^{-j \omega(t)\left(t-\tau_{\mathrm{R}}\right)} \boldsymbol{R}_{\mathrm{R}, s} \boldsymbol{R}_{\mathrm{R}}(\omega) \boldsymbol{p}_{\mathrm{STL}} \cdot \boldsymbol{s},
$$

where $A \mathrm{e}^{-j \omega(t)\left(t-\tau_{\mathrm{R}}\right)} \boldsymbol{p}_{\mathrm{STL}}$ is the field with complex amplitude $A$ and angular frequency $\omega$ generated by the swept tunable laser (STL) at the input of the polarization diverse receiver. Parameter $p_{S T L}$ denotes the polarization state at the source, $\sqrt{\frac{1-\kappa_{1}}{2}}, \sqrt{\frac{\kappa_{2}}{2}}$ and $\frac{1}{2}$ stem from the split in reference and device arm, split off of the clock interferometer and the split of the reference at the polarization diverse receiver respectively. Therein the fading becomes immediately obvious as

$$
0 \leq \boldsymbol{R}_{\mathrm{R}, s} \boldsymbol{R}_{\mathrm{R}}(\omega) \boldsymbol{p}_{\mathrm{STL}} \cdot \boldsymbol{s} \leq 1,
$$

which directly affects the generated photocurrent on the balanced photodetector, which is proportional to the reference signal as

$$
i_{s} \propto\left|\boldsymbol{E}_{\mathrm{D}, s}\right|\left|\boldsymbol{E}_{\mathrm{R}, s}\right| \propto \boldsymbol{R}_{\mathrm{R}, s} \boldsymbol{R}_{\mathrm{R}}(\omega) \boldsymbol{p}_{\mathrm{STL}} \cdot \boldsymbol{s}
$$




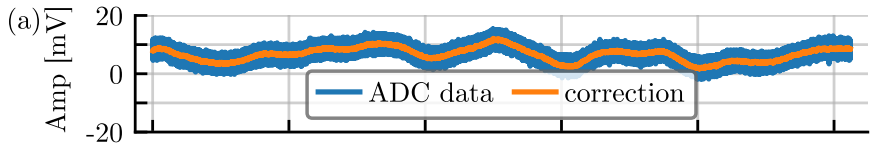

(b)

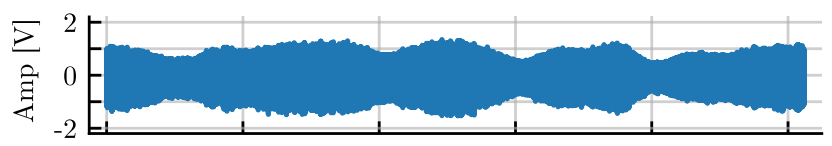

(c)

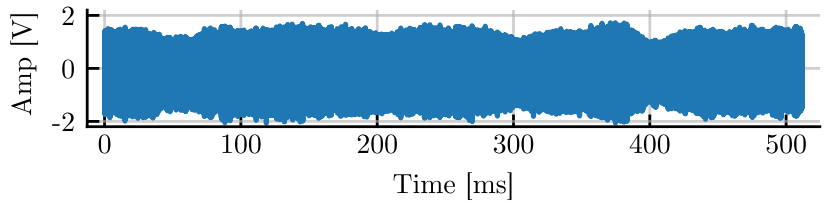

Fig. 2. Sample waveforms of the polarization equalization process: (a) polarization reference waveform and the correction derived from it; (b)-(c) one of the two outputs of the polarization diverse receiver before (b) and after (c) polarization equalization.

\section{OVNA REFERENCE ARM POLARIZATION EQUALIZATION}

The proposed polarization equalization scheme is based on polarizing the light of the reference arm and tapping a small fraction to serve as reference for correction of the fading seen in Eq. (2), arising from the variation of polarization with wavelength. To this end, a polarizer is included in the reference arm, after the fiber delay $\tau_{r}$, as highlighted in Fig. 1. The polarized reference signal is then combined with the measurement signals for detection using balanced photodetectors. The power fading introduced by the polarizer is captured by redirecting a fraction of the polarized reference signal to a conventional single-ended photodiode. The waveform is digitized by the same analog-to-digital converter (ADC) as the two data channels and the reference clock for sweep linearization. The polarization reference signal recorded in this fashion can directly serve for polarization equalization, as the fading observed in the beating of the signals from the device and reference arms on the balanced photo-detectors (BPDs) of the polarization diverse receiver is inversely proportional to the power of the signal in the reference arm.

The actual polarization equalization is performed digitally. Figure 2, shows waveforms of the polarization tracking signal and the derived equalization signal (Fig. 2(a)) and one of the measurement channels before equalization (Fig. 2(b)). A similar variation over time can be seen for both waveforms. Note that as result of the laser sweep, the wavelength varying behaviour translates to a time-varying effect.

A moving average filter is applied to the polarization tracking data to obtain the slowly-varying correction signal, represented by the orange curve in Fig. 2(a). From this correction signal, any DC offset resulting from the ADC frontend is removed, before it is normalized to yield unitary gain at its maximum - based on the assumption of the incoming reference polarization aligning with the polarizer at least once during the sweep. Subsequently, the inverse of the signal is applied to the measurement data of Fig. 2(b), resulting in the equalized waveform depicted in Fig. 2(c). Note that the wavelength varying fading is reduced and as a result, the average signal power is increased. The two trenches around $60 \mathrm{~ms}$ and $400 \mathrm{~ms}$, although strongly reduced, still remain in the final waveform, indicating imperfect equalization. This is attributed to the fact that at these timestamps the polarization tracking signal is close to 0 (after DC removal) and therefore the algorithm struggling to apply the right, i.e., sufficiently large, correction.

After equalization, the signals are treated analog by the signal processing chain shown in Fig. 1, analog to the discussion in [10]-[13]. That is, first the two outputs from the polarization diverse receiver are resampled to a linear frequency grid obtained from the fringe pattern produced by the reference clock interferometer. Then, in their spectra, the impulse response elements of the DUT appear at frequencies related to the fiber delay lines applied at the in- and output ports of the device. Finally the transfer function matrix is calculated via inverse fast Fourier transform (IFFT), and linear device parameters are derived by singular value decomposition (SVD). The IL - used in the next section as a measure to show the effectiveness of polarization equalization - is calculated at every wavelength as the mean of the singular values squared of the transfer function matrix.

\section{INSERTION LOSS ANALYSIS OF 39-CORE FM-MCF}

In order to demonstrate the polarization equalization, a large core count FM-MCF is analyzed. The fiber hosts 38 fewmode cores, and 1 single-mode core targeted for self coherent detection schemes [4]. For the analysis with the OVNA setup depicted in Fig. 1, the single-mode core is employed as the reference arm [12], [14], [15]. The polarization, input, and output delays are configured with a minimum spacing of $\tau=4.5 \mathrm{~ns}$. The STL is swept from $1530 \mathrm{~nm}$ to $1570 \mathrm{~nm}$ at $100 \mathrm{~nm} / \mathrm{s}$ with $12 \mathrm{dBm}$ output power. The core multiplexer and de-multiplexer are fusion spliced to $13.6 \mathrm{~km}$ of FM-MCF, and the same pair of single-core, three-mode, non-mode-selective photonic lanterns (PLs) are swapped between the input and output few-mode fibers of the core (de-)multiplexers. The signal from the DUT is combined with the reference arm and detected in a polarization diverse receiver with BPDs (Thorlabs PDB435C). The combined power on each BPD is on the order of $-10 \mathrm{dBm}$ and the resulting interferograms are sampled at $10 \mathrm{MSa} / \mathrm{s}$ with 8 bit resolution.

For each core the $6 \times 6$ transfer function matrix is measured and the singular values are calculated at each measured frequency. Subsequently the IL is calculated in each $50 \mathrm{GHz}$ subband to allow easier illustration. The average IL for each core is given in Fig. 3(a). Note that the lowest IL (with exception of the single-mode core) is observed for the center core, which is the least sensitive to rotational misalignment. Furthermore, cores $10,14,15,25$ and 32 have significantly larger losses, which is in agreement with previous measurements [4]. The other cores are close to the average IL of $8.7 \mathrm{~dB}$.

The wavelength dependent fluctuation of the IL of each core is shown in Fig. 3(b), where the color scale represents the deviation from the mean IL of each core. The dashed lines 


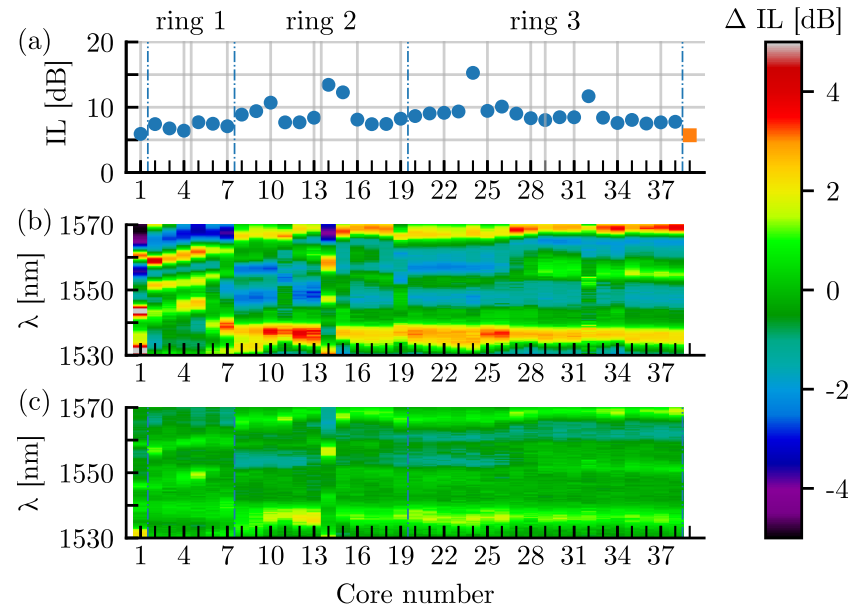

Fig. 3. Average core IL for the 39 fiber cores (a) and wavelength variation of IL after subtracting core average IL without polarization equalization (b), and with polarization equalization applied (c).

mark the 4 concentric rings on which the cores are positioned in the FM-MCF. Figure 3(c) depicts the same data, but with the polarization equalization enabled in the first part of the DSP sequence. A significant reduction of variation of IL across wavelength is observed. Without polarization equalization, the standard deviation of the IL is found to be 1.48, while it is reduced to 0.70 for the equalized signals. Note that cores on the outer two rings $(\geq 8)$ have increased losses around $1540 \mathrm{~nm}$ and $1570 \mathrm{~nm}$. Translating these back to sweep time, these points correspond to the lowest signal power points of the polarization tracker signal in Eq. (2)(a), where, as previously discussed, the equalization scheme struggles to fully equalize the deep fading. It is expected that this issue could be partially resolved by applying additional power to the polarization reference (at the cost of power in the reference arm) or through the use of a high sensitivity photodiode. The introduction of a polarization tracker/controller able to follow at close to the speed of the observed rotations could prevent deep fades, while remaining transients from polarization tracking would be eliminated by the proposed method; similarly a construction based on Faraday mirrors might alleviate the issue at the cost of additional system complexity [16].

Finally, note that the wavelength dependent and average IL for core 14 differs strongly from the other cores and for this core a higher error vector magnitude (EVM) was found during transmission [4].

\section{Conclusions}

In this work, the problem of wavelength dependent polarization of the reference signal in optical vector network analysis has been discussed, a simple equalization method proposed, and experimentally validated in the measurement of a 39-core FM-MCF. Specifically, the analysis of longer stretches of SDM fibers requires that the length of the reference arm of the OVNA is matched to the length of the fiber under test. However, the inclusion of additional fiber in the reference arm leads to a variation of the polarization over wavelength. This results in fading of the signals detected by the OVNA which significantly distort the measured transfer function matrix compared to the case when the DUT length is small, i.e., a few meters of fiber.

The proposed technique is based on the introduction of a polarizer in the reference arm of the OVNA and the acquisition of a fraction of the resulting signals as polarization fading reference signal. Applying the inverse of this signal, it has been shown that the fading can be compensated and the measurement distortions significantly reduced. This equalization technique has been demonstrated by measuring the insertion loss of a 39-core FM-MCF, significantly improving the measurements of wavelength dependent IL and considerably reducing the measurement error due to fading induced by the wavelength dependency of the polarization. Moreover, the proposed technique constitutes a small but fundamental step to improve OVNA measurements of long lengths of SDM fibers, hence contributing to the further advancement and timely adoption of SDM in future commercial systems.

\section{REFERENCES}

[1] D. J. Richardson, J. M. Fini, and L. E. Nelson, "Space-division multiplexing in optical fibres," Nat. Photon., vol. 7, pp. 354-362, Apr. 2013.

[2] P. J. Winzer and D. T. Neilson, "From scaling disparities to integrated parallelism: A decathlon for a decade," J. Lightw. Technol., vol. 35, no. 5, pp. 1099-1115, Mar. 2017.

[3] D. Soma et al., "10.16-Peta-bit/s dense SDM/WDM transmission over 6-mode 19-core fiber across the C+L band," J. Lightw. Technol., vol. 36, no. 6, pp. 1362-1368, Mar. 2018.

[4] J. Sakaguchi et al., "228-spatial-channel bi-directional data communication system enabled by 39-core 3-mode fiber,' J. Lightw. Technol., vol. 37, no. 8, pp. 1756-1763, Apr. 2019.

[5] J. M. Kahn and K.-P. Ho, "Linear propagation effects in mode-division multiplexing systems," J. Lightw. Technol., vol. 32, no. 4, pp. 614-628, Feb. 2014

[6] R. Maruyama, N. Kuwaki, S. Matsuo, and M. Ohashi, "Relationship between mode coupling and fiber characteristics in few-mode fibers analyzed using impulse response measurements technique," J. Lightw. Technol., vol. 35, no. 4, pp. 650-657, Feb. 2017.

[7] T. Arakawa, F. Ito, D. Iida, and T. Manabe, "Impulse response of weaklycoupled multicore fiber measured by using high-dynamic-range linear optical sampling," Opt. Fiber Technol., vol. 50, pp. 188-193, Jul. 2019.

[8] G. D. VanWiggeren and D. M. Baney, "Swept-wavelength interferometric analysis of multiport components," IEEE Photon. Technol. Lett., vol. 15, no. 9, pp. 1267-1269, Sep. 2003.

[9] D. K. Gifford, B. J. Soller, M. S. Wolfe, and M. E. Froggatt, "Optical vector network analyzer for single-scan measurements of loss, group delay, and polarization mode dispersion," Appl. Opt., vol. 44, no. 34, pp. 7282-7286, Dec. 2005.

[10] N. K. Fontaine et al., "Characterization of space-division multiplexing systems using a swept-wavelength interferometer," in Opt. Fiber Commun. Conf. (OFC). OSA, Mar. 2013, paper OW1K.2.

[11] S. Rommel et al., "Few-mode fiber, splice and sdm component characterization by spatially-diverse optical vector network analysis," Opt. Express, vol. 25, no. 19, pp. 22 347-22 361, Sep. 2017.

[12] J. J. A. van Weerdenburg et al., "Enhanced modal dispersion estimation enabled by chromatic dispersion compensation in optical vector network analysis," J. Lightw. Technol., vol. 37, no. 16, pp. 4001-4007, Aug. 2019.

[13] B. Heffner, "Automated measurement of polarization mode dispersion using Jones matrix eigenanalysis," IEEE Photon. Technol. Lett., vol. 4, no. 9, pp. 1066-1069, Sep. 1992.

[14] S. Rommel et al., "Measurement of modal dispersion and group delay in a large core count few-mode multi-core fiber," in European Conf. Optical Communication (ECOC). IEEE, Sep. 2018, paper Tu1E.3.

[15] J. J. A. van Weerdenburg et al., "Chromatic dispersion analysis and compensation in a large core-count few-mode multi-core fiber based on optical vector network analysis," in Opt. Fiber Commun. Conf. (OFC). OSA, Mar. 2019, paper M1E.2.

[16] A. D. Kersey, M. J. Marrone, and M. A. Davis, "Polarisation-insensitive fibre optic Michelson interferometer," Electron. Lett., vol. 27, no. 6, pp. 518-520, Mar. 1991. 